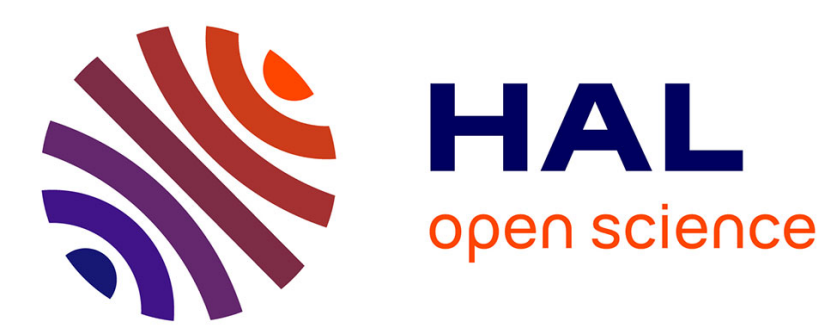

\title{
A valued Ferrers relation for interval comparison
}

Meltem Öztürk, Alexis Tsoukias

\section{To cite this version:}

Meltem Öztürk, Alexis Tsoukias. A valued Ferrers relation for interval comparison. Fuzzy Sets and Systems, 2015, 266, pp.47-66. 10.1016/j.fss.2014.09.004 . hal-02787810

\section{HAL Id: hal-02787810 https://hal.science/hal-02787810}

Submitted on 30 Nov 2020

HAL is a multi-disciplinary open access archive for the deposit and dissemination of scientific research documents, whether they are published or not. The documents may come from teaching and research institutions in France or abroad, or from public or private research centers.
L'archive ouverte pluridisciplinaire HAL, est destinée au dépôt et à la diffusion de documents scientifiques de niveau recherche, publiés ou non, émanant des établissements d'enseignement et de recherche français ou étrangers, des laboratoires publics ou privés. 


\title{
A valued Ferrers relation for interval comparison
}

\author{
Meltem Öztürk, Alexis Tsoukiàs \\ \{ozturk, tsoukias\}@lamsade.dauphine.fr \\ tel : +33144054419 \\ fax: +33144054091 \\ CNRS-Lamsade, Université Paris Dauphine, Paris, France
}

\begin{abstract}
The paper deals with the valued comparison of intervals for decision making. Interval orders are classical preference structures where the comparison of intervals is done in an ordinal way. In this paper we focus on valued comparison where more information, especially the distance between end-points of intervals, is used in order to have more sophisticated preference structures. The generalization of an interval order as a valued structure requires the choice of de Morgan triplets. We propose a valued outranking relation for interval comparison and show that it satisfies different definitions of valued interval orders using different de Morgan triplet. The decomposition of our outranking relation into preference and indifference provides a valued preference structure where the preference is $T$-transitive and monotone.
\end{abstract}

Key words: decision making, preference modeling, valued binary relation, interval comparison, interval orders, Ferrer relation

\section{Introduction}

Most decision making problems in concrete applications are situated in complex environments where one needs to take into consideration incomplete or imprecise knowledge, and/or vague preferences. When intervals are used in order to represent such type of information, the decision maker needs to define a preference structure that allows to establish a preference between objects represented by intervals. In this article we propose comparison rules on intervals in form of valued binary relations and analyze the properties of these relations from a preference modeling point of view. Preference modeling, field where preference relations are studied in details, is a central step for decision aiding but it is also 
commonly used in other domains such as artificial intelligence, economy, political sciences, psychology and sociology. Interested reader can find more details in $[3,10,11,15,16,17]$ among many others.

In preference modeling, intervals are used as numerical representation of some well known preference structures such as semiorders, interval orders, $P Q I$ interval orders, pseudo orders, etc. (see $[10,16,18])$. The numerical representation of preferences is a widely studied topic since the quantification of preferences facilitates the search of the best object(s). In this article we restrict our study to the case of a finite set of objects knowing that the cardinality of the object set plays an important role on the representation. We should mention the case of preference structures applied on infinite sets where topological structures are required (see $[4,13]$ for relations between orders and topology and $[1,2]$ for continuous representation of IO). In decision making, a numerical representation of a preference structure may be used for two different purposes. Let us consider the case of a preorder.

Definition 1.1. (Preorder) Let $R$ be a binary relation on $A \times A$, then $R$ is a preorder if and only if there exists a real valued function $f$ such that $\forall x, y \in$ $A R(x, y) \Longleftrightarrow f(x) \geq f(y)$.

Imagine now that the way in which the decision maker judges the objects corresponds to the logic of a preorder. The part $\forall x, y \in A R(x, y) \Longrightarrow f(x) \geq f(y)$ (right implication) of the definition may be used when the pairwise comparisons on objects are known and when the decision maker needs to associate a number to each object. The part $\forall x, y \in A f(x) \geq f(y) \Longrightarrow R(x, y)$ (left implication) may be used when the evaluation of each object is known and when a ranking on objects is needed.

In our point of view, the use of intervals in the numerical representation of preference structures is mostly related to the right implication of the characterization. Intervals are useful since some type of intransitivity of indifference (generally related to the cumulation of small indifference thresholds) may be represented by intervals but not by single points. Concerning the left implication, the use of the definition of such preference structures in order to compare intervals of reals is generally limited. The mean reason is the fact that these definitions make use of ordinal comparisons which means that the distances between the points of intervals are not important and that we can get several different numerical representations. Hence when the comparison rules of semiorders or interval orders are used in real life problems some information is lost. For instance, consider three 
intervals $x=[0,6], y=[1,5]$ and $z=[5,10]$. For interval orders, intervals $x$ and $y$ are indifferent but also $x$ and $z$ are indifferent.

The aim of our study is to propose some comparison rules for intervals where the distance between points will be significant. We want to have intuitive and easily interpretable rules (which can facilitate their acceptability by decision makers) but we want also that the resulting valued preference relations have some nice properties as it is the case in crisp interval orders.

The fuzzification of interval orders is already studied in a theoretical way where the researchers analyzed different possible ways to define them $([5,7])$. However a comparison rule of intervals satisfying such definitions is not proposed in the literature. In this article we propose valued binary relations on intervals, show that they satisfy some nice properties and that they can be seen as a fuzzification of interval orders.

The paper is organized as follows. In the next section we introduce the basic notations, Section 3 is devoted to the definitions of valued interval orders where we put together some results of $[5,7]$. In Section 4 we present a valued outranking relation for the comparison of intervals and study its properties. We show that our valued relation satisfies different definitions of fuzzy interval orders. In Section 5 we divide our outranking relation into its asymmetric and symmetric parts in order to differentiate the preference from the indifference and analyze the properties of these two relations. We show that our relations form a valued preference structure with some nice properties and we conclude the paper with Section 6.

\section{Notations and definitions}

In preference modeling the comparison of objects is done by binary relations. In this paper $A$ will represent a finite set of elements on which preference relations are applied. We will use capital letters $P, Q, R, \ldots$ to represent the relations as subsets of $A \times A$, thus in crisp case the notation $R(x, y)$ and $x R y$ are used indifferently and they show that the relation $R$ holds among $x$ and $y$. Given any two binary relations $V, W$ on the set $A$ we denote $V \circ W(x, y)$ if and only if $\exists z \in A: V(x, z) \wedge W(z, y)$ and $V \subseteq W$ is the formula $\forall x, y V(x, y) \Rightarrow W(x, y)$.

The collection of binary relations used for preference modelling is called a preference structure. In general the classical theory of preference modeling considers two relations, the preference relation $(P)$ and the indifference relation $(I)$. Such a representation admits the existence of a complete preference structure, i.e. the decision maker is supposed to be able to compare the alternatives (for all object $x$ and $y$ in $A, x P y$ or $y P x$ or $x I y$ holds) in an exclusive way $(P \cap I=\emptyset)$. 
The collection of $P$ and $I$ is called a $\langle P, I\rangle$ preference structure.

Definition 2.1. ( $\langle P, I\rangle$ Preference structure [18]) Let $P$ and I be two binary relations defined on $A \times A$, then they form a $\langle P, I\rangle$ preference structure iff

- $P$ is irreflexive, $I$ is reflexive

- $P$ is asymmetric

- I is symmetric

- $P \cap I=\emptyset$ (exclusivity)

- $P \cup I \cup P^{-1}=A \times A$ (exhaustivity) $\left(\forall x, y P^{-1}(x, y)=P(y, x)\right)$

The union of preference and indifference is generally called the outranking relation or the characteristic relation. The affirmation $x R y$ holds if and only if " $x$ is at least as good as $y$ ". The indifference relation (resp. the preference relation) is the symmetric (resp. the asymmetric) part of $R$. From a data analysis point of view we can consider the indifference relation as a similarity relation and the union of the preference relation and its inverse as a dissimilarity relation. Hereafter, for sake of simplicity, we will only use the terms of preference and indifference

In the following we will consider objects represented under form of intervals of values. Given a finite set $A$ of objects we associate to each element of $A$ two functions $l: A \mapsto \mathbb{R}$ and $u: A \mapsto \mathbb{R}$ where $l(x)$ (resp. $u(x)$ ) represents the left (resp. the right) end-point of the interval of the object $x$; hence we suppose that for all $x$ in $A, l(x)<u(x)$. Given the finite structure of set $A$, when we compare intervals we can restrict inequalities to their strict part without loss of any generality.

Studying valued relations needs some notions of fuzzy set theory. Small letters $p, q, r, \ldots$ are used to represent the relations as predicates holding among two elements of $A$ in the fuzzy case (for instance $p(x, y)$ will represents the intensity or the credibility of the preference of $x$ over $y$ ). We are going to use the symbols of $T$ and $S$ in order to represent t-norms (triangular norm) and t-conorms (triangular conorm) respectively as continuous representations of conjunction and disjunction operators in the case of continuous valuations.

Definition 2.2. (Triangular norm [19]) A triangular norm $T$ is an increasing, associative and commutative $[0,1]^{2} \longrightarrow[0,1]$ mapping satisfying $\forall x \in[0,1]$, $T(1, x)=x$. 
Definition 2.3. (Triangular conorm [19]) A triangular conorm $S$ is an increasing, associative and commutative $[0,1]^{2} \longrightarrow[0,1]$ mapping satisfying $\forall x \in[0,1]$, $S(0, x)=x$.

T-norms and t-conorms are used for the definition of intersection and union: the fuzzy intersection $r \cap_{T} b$ is the binary fuzzy relation defined by $\forall x, y r \cap_{T}$ $b(x, y)=T(r(x, y), b(x, y))$; the fuzzy union $r \cup_{S} b$ is the binary fuzzy relation defined by $\forall x, y r \cup_{S} b(x, y)=S(r(x, y), b(x, y))$.

Definition 2.4. (Negation) A negation $N$ is a function from $[0,1]$ to $[0,1]$ which satisfies the following conditions:

- $N(0)=1$ and $N(1)=0$

- $\forall x, y x \leq y \Longrightarrow N(y) \leq N(x)$

A continuous and strictly decreasing negation is called a strict negation. In this paper, if nothing is precised, we will refer to the standard negation which is $\forall x \in[0,1], N(x)=1-x$ (for a discussion about such operators in the frame of fuzzy sets theory see [9]).

T-norms and t-conorms are related by duality. Pairs of t-norms and t-conorms satisfy the generalisation of the De Morgan law as in the following:

Definition 2.5 (De Morgan Triplets ). Let $T$ be a t-norm, $S$ a t-conorm and $N$ a strict negation. Then $\langle T, S, N\rangle$ is a De Morgan triplet iff $\forall x, y \in[0,1]$ :

$$
N(S(x, y))=T(N(x), N(y)) .
$$

Several De Morgan Triplets have been suggested in the literature. Zadeh (called also Gödel) and Łukasiewicz triplets are the most used ones in preference modeling (see Table 2).

\begin{tabular}{lcc}
\hline Names & $t$-norms & $t$-conorms \\
\hline Zadeh & $\min (x, y)$ & $\max (x, y)$ \\
Łukasiewicz & $\max (x+y-1,0)$ & $\min (x+y, 1)$ \\
\hline
\end{tabular}

Table 1: De Morgan triplets

Some properties may be important for the use of t-norms, we present in the following two of them which are necessary for the analysis of valued preference structures. 
Definition 2.6. (Zero divisor [14]) An element $x \in] 0,1[$ is called a zero divisor of a t-norm $T$ iff $\exists y \in] 0,1[$ such that $T(x, y)=0$. A t-norm without zero divisors is called positive.

Definition 2.7. (Archimedean [14]) A continuous t-norm $T$ is Archimedean iff $\forall x \in] 0,1[T(x, x)<x$.

Lukasiewicz t-norm is Archimedean and has zero-divisors while the minimum operator is positive.

One of the main topic of preference modeling is the analysis of the properties of preference relations. We present in the following some definitions of properties in the valued case:

Definition 2.8. Considering a t-norm $T$ and a $t$-conorm $S$, a binary valued relation $r$ in $A \times A$ is

- reflexive if and only if $\forall x, r(x, x)=1$

- irreflexive if and only if $\forall x, r(x, x)=0$

- symmetric if and only if $\forall x, y r(x, y)=r(y, x)$

- $T$-asymmetric if and only if $\forall x, y, T(r(x, y), r(y, x))=0$

- $T$-antisymmetric if and only if $\forall x, y, x \neq y \Rightarrow T(r(x, y), r(y, x))=0$

- $S$-strong complete if and only if $\forall x, y, S(r(x, y), r(y, x))=1$

- S-complete if and only if $\forall x, y, x \neq y \Rightarrow S(r(x, y), r(y, x))=1$

- T-transitive if and only if $\forall x, y, z, T(r(x, y), r(y, z)) \leq r(x, z)$

Strong completeness implies the reflexivity in the crisp case however in the valued case one can have a strong complete relation $r$ which does not satisfy the reflexivity, it is the case, for instance, if the strong completeness is verified using the Łukasiewicz t-conorm.

The dual of a valued relation $r$ on $A$ is denoted by $r^{d}$ and defined by $\forall x, y \in$ $A, r^{d}(x, y)=N(r(y, x))$. An implication using a composition of two valued relations such as $v \circ_{T} w \subseteq q$ will be represented as $\forall x, y, z \in A, T(v(x, y), w(y, z)) \leq$ $q(x, z)$. 
Total orders (transitive, antisymmetric and complete relations) and preorders (reflexive, transitive and complete) are the most used preference structures in decision aiding. Their definition in valued case is derived from their definition in crisp case:

Definition 2.9. (Valued total order [11]) Considering a $t$-norm $T$ and a $t$-conorm $S$, a binary valued relation $r$ is a valued total order if and only if $r$ is, $T$-antisymmetric, S-strong complete and T-transitive.

Definition 2.10. (Valued preorder [11]) Considering a t-norm $T$ and a $t$-conorm $S$, a binary valued relation $r$ is a valued preorder if and only if $r$ is reflexive, $S$ strong complete and T-transitive.

In the crisp case, in both of these structures, a small difference between two evaluations is interpreted as a preference for the object having better evaluation. However, as pointed by Luce ([12]), in some situations small differences may be considered as not significative for decision makers. Imagine, for instance, a decision maker looking for a new car and comparing two cars with price equal to $20000 €$ for the first one and price equal to $20500 €$ for the second one. It is not surprising if the decision maker says that he is indifferent between these two prices. Such an idea of indifference threshold can not be handled by total orders or preorders. Interval orders are used in such situations. The characterization of interval orders in the valued case needs special analysis, we devote the following section to this topic.

\section{Fuzzy Interval orders}

We start by presenting the definition of an interval order in discrete case:

Definition 3.1. (Interval order [10]) Let $R$ be a binary relation on $A \times A$, then $R$ is an interval order if and only if $R$ is complete and Ferrers.

Definition 3.2. (Ferrer relation [10]) Let $R$ be a binary relation on $A \times A$, then $R$ is Ferrers if and only if

$$
\forall x, y, z, w \in A, \quad(x R y \wedge z R w) \Longrightarrow(x R w \vee z R y)
$$

A Ferrer relation can be also defined using the dual of $R$. 
Proposition 3.1. ([10]) Let $R$ be a binary relation on $A \times A$, then $R$ is Ferrers if and only if

$$
R \circ R^{d} \circ R \subseteq R
$$

As we mentioned in Section 2 an outranking relation can be divided in two parts, the asymmetric part denoted by $P$ and the symmetric one denoted by $I$. The name of interval orders is inspired by the fact that the numerical representation of these structures makes use of intervals.

Definition 3.3. (Interval order [10]) $A\langle P, I\rangle$ preference structure on a set $A$ is an interval order if and only if $\exists l, u: A \mapsto \mathbb{R}$ such that:

$\forall x: u(x) \geq l(x)$;

$\forall x, y: P(x, y) \Leftrightarrow l(x)>u(y)$

$\forall x, y: I(x, y) \Leftrightarrow l(x) \leq u(y)$ and $l(y) \leq u(x)$.

A representation with $l$ and $u$ is equivalent to the one where to each element $x$ of $A$ is associated a function $g(x)$ (representing the utility of $x$ ) and a threshold function $t(x)$ where we have $l(x)=g(x)$ and $u(x)=g(x)+t(x)$. In this case one will say that the object $a$ is preferred to the $b$ if and only if its utility is greater than the utility of $b$ plus an indifference threshold.

The special case where the length of intervals are constant (hence the indifference threshold is constant) corresponds to a semiorder.

Using the properties of $P$ and $I$, one can provide new ways to define an interval order. In the following we present four equivalent definitions for these structures.

Theorem 3.1. [10] Let $R$ be the characteristic relation of a the $\langle P, I\rangle$ preference structure on a set $A$, the following assertions are equivalent:

i. $R$ is an interval order.

ii. $\forall x, y, z, w \in A(x R y \wedge z R w) \Longrightarrow(x R w \vee z R y)$.

iii. $R \circ R^{d} \circ R \subseteq R$.

iv. $P \circ P^{d} \circ P \subseteq P$.

v. $P \circ I \circ P \subseteq P$. 
All the equivalences between these five conditions do not hold in the fuzzy case ([8]). As a consequence different types of fuzzy interval orders may be defined in the valued case. Condition (ii) is the most used one but other conditions are also suggested by different researchers as alternative definitions. Bufardi, Van de Walle and De Baets have analyzed relations between these definitions and defined equivalence classes (see [5, 7]. Some new results on the approach of [7] can be found in [8]).

The names of our definitions are different from the name given by Bufardi, Van de Walle and De Baets. We consider in the following a reflexive and $S$-complete (the completeness must be verified with any t-conorm) relation $r$. Concerning relations $p$ and $i$, their union is also supposed to be reflexive and $S$-complete; $p$ and $i$ form a fuzzy preference structure.

- The direct use of the definition of fuzzy Ferrers relation ([7]):

- $r$ is a min-interval order iff $\forall x, y, z, t \in A$,

$$
T_{\min }(r(x, y), r(z, t)) \leq S_{\max }(r(x, t), r(z, y)) .
$$

- $r$ is a Łukasiewicz-interval order iff $\forall x, y, z, t \in A$

$$
T_{L}(r(x, y), r(z, t)) \leq S_{L}(r(x, t), r(z, y))
$$

- The use of the fuzzy composition of $p$ and $i$ ([7]):

- $p$ and $i$ form a strong-interval order iff

$$
p \circ_{T_{\min }} i \circ_{T_{\min }} p \subseteq p .
$$

- $p$ and $i$ form a weak-interval order iff

$$
p \circ_{T_{L}} i \circ_{T_{L}} p \subseteq p .
$$

- The use of the fuzzy composition of $r$ and $r^{d}([5])$ :

- $r$ is a $T_{L}-S_{L}$-interval order iff

$$
r \circ_{T_{L}} r^{d} \circ_{T_{L}} r \subseteq r
$$

- The use of $\lambda$-cuts of $r([5,7])$ : 
- $r$ is a min-max-interval order iff $\forall i \in[0,1]$,

$R_{\lambda_{i}}$ is Ferrers(i.e. $R_{\lambda_{i}}$ is an interval order, see Definition 3.1)

where $R_{\lambda_{i}}=\left\{(x, y), r(x, y) \geq \lambda_{i}\right\}$,

- $p$ and $i$ form a $\lambda$-interval order iff $\forall i \in[0,1]$,

$$
P_{\lambda_{i}} \cup I_{\lambda_{i}} \text { is Ferrers. }
$$

where

$P_{\lambda_{i}}(x, y) \Longleftrightarrow r(x, y) \geq \lambda_{i}$ and $r(y, x)<\lambda_{i}$ and

$I_{\lambda_{i}}(x, y) \Longleftrightarrow \min (r(x, y), r(y, x)) \geq \lambda_{i}$.

Although these definitions are not always equivalent, there exist some relations between them. We present in the following a general scheme showing such relations between different definitions. For this purpose we put together in Figure 1 different results obtained by the three authors (see $[5,7]$ ) and add two counter examples on these results in order to complete the scheme.

Theorem 3.2. The classes of fuzzy preference structures defined by each row of Figure 1 are identical within each box. The box classes are partially ordered by inclusion from bottom to top according to the arrows. The absence of an arrow means that the inclusion is not verified.

Proof : see Annexe (Section 7).

\section{Comparison of intervals with a valued Ferrers relation}

As we explained in the introduction, the aim of our article is to propose some comparison rules on intervals with some nice properties from preference modeling point of view. Moreover, we want our rules to have a semantic easy to understand for decision makers. Hence, we begin our study by proposing some ideas about what the outranking relation must contain. We think that there are some positive reasons to affirm that the interval $x$ is at least as good as the interval $y$ if:

i. some points of $x$ are greater than all the points of $y$ or

ii. some points of $y$ are smaller than all the points of $x$ or

iii. $x$ and $y$ have some common points. 
1) $T_{\min }(r(x, y), r(z, t)) \leq S_{\max }(r(x, t), r(z, y))$

2) $T_{\min }(p(x, y), p(z, t)) \leq S_{\max }(p(x, t), p(z, y))$

3) $\forall \lambda R_{\lambda}$ Ferrers

4) $\forall \lambda\left(P_{\lambda}, I_{\lambda}\right)$ Ferrers

5) $p \circ_{T_{\min }} i \circ_{T_{\min }} p \subseteq p$

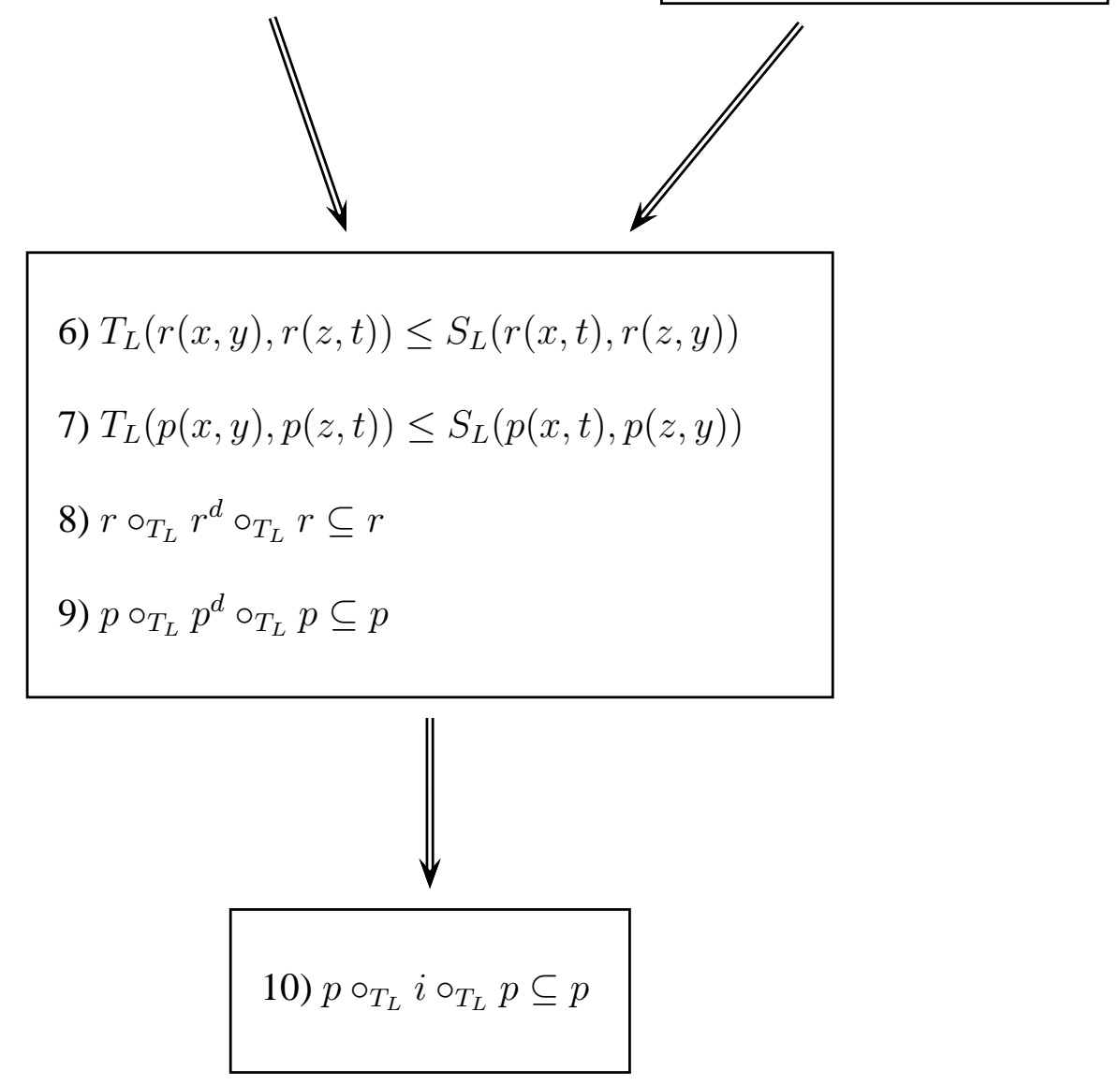

Figure 1: Relations between different definitions of fuzzy interval orders. 
These ideas may be represented by the proportion of points satisfying the conditions cited above :

i. the proportion of points of interval $x$ which are greater than all the points of interval $y: \min \left(1, \max \left(\frac{u(x)-u(y)}{u(x)-l(x)}, 0\right)\right)$,

ii. the proportion of points of interval $y$ which are smaller than all the points of interval $x: \min \left(1, \max \left(\frac{l(x)-l(y)}{u(y)-l(y)}, 0\right)\right)$,

iii. the proportion of points of interval $x$ which are also points of interval $y$ : $\min \left(1, \max \left(\frac{x \cap y}{u(x)-l(x)}, 0\right)\right)$.

where $x \cap y$ presents the difference $(\min (u(x), u(y))-\max (l(x), l(y)))$.

Hence we propose to define the outranking relation, denoted by $r$, as the sum of these three proportions.

Definition 4.1. (Outranking relation for intervals) Let $r$ be a valued binary relation on $A \times A$ then it is an outranking relation for intervals iff $\forall x, y$

$$
\begin{array}{r}
r(x, y)=\min \left(1, \max \left(\frac{u(x)-u(y)}{u(x)-l(x)}, 0\right)+\max \left(\frac{l(x)-l(y)}{u(y)-l(y)}, 0\right)+\right. \\
\left.\max \left(\frac{x \cap y}{u(x)-l(x)}, 0\right)\right) .
\end{array}
$$

Table 2 represents the value of $r(x, y)$ with respect to different relative positions of $x$ and $y$. This table presents all the possibilities on relative positions in an exhaustive way, the cases where one end-point of $x$ meets one end-point of $y$ correspond to two consecutive cases, for instance the case $l(x)=u(x)<u(y)<$ $u(x)$ corresponds to the case 2 and 3 .

We will analyse in the following different properties of $r$. We begin by showing that $r$ is reflexive and $S$-strong complete.

Proposition 4.1. Let $r$ be the valued outranking relation of Definition 4.1, then $r$ is reflexive and $S$-strong complete.

Proof : see Annexe (Section 7).

Another property which is important for preference comparison is the monotonicity. This property can be obtained by imposing $r$ to be non decreasing on the first element and non increasing on the second one. 


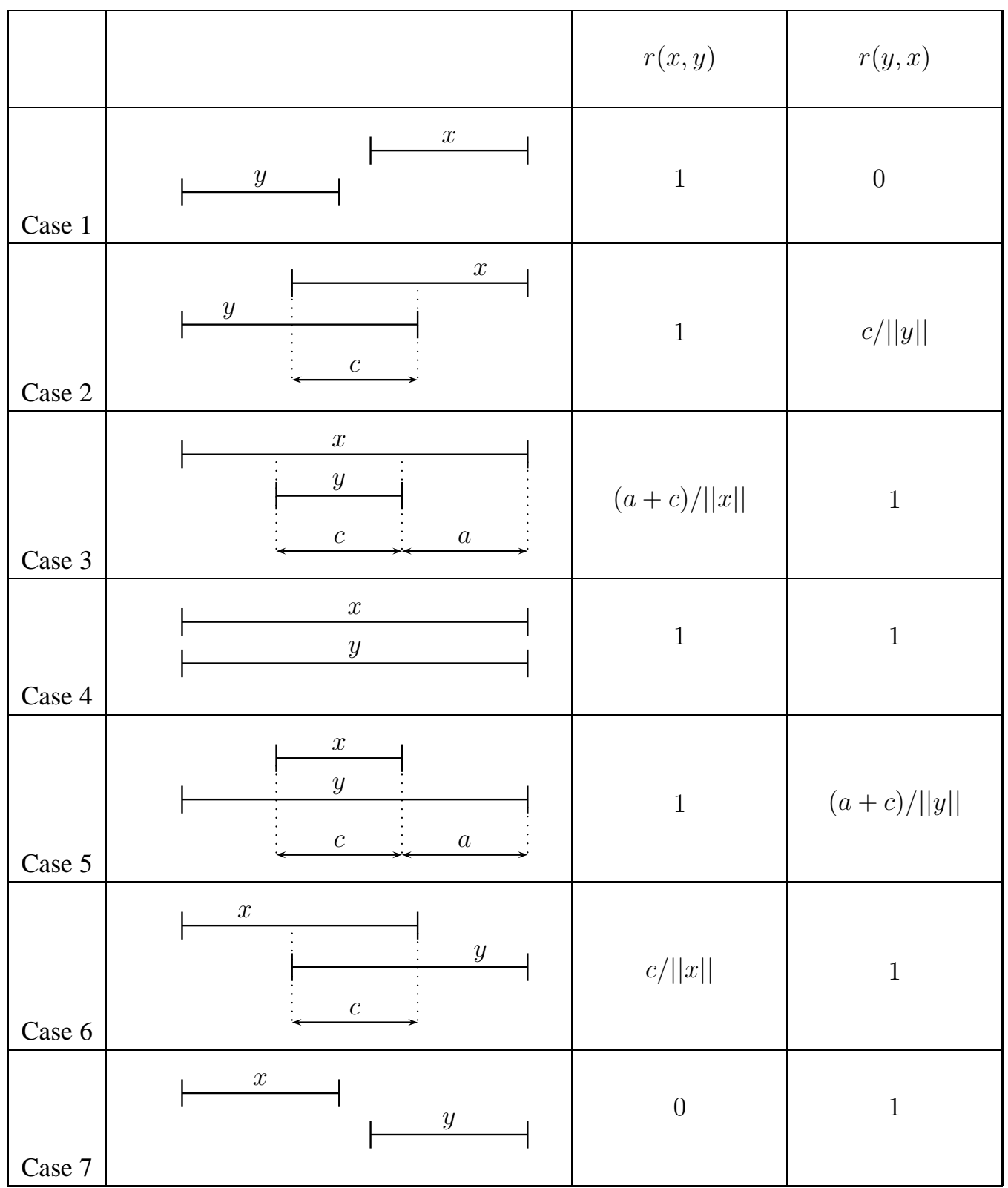

Table 2: Different values of the outranking relation for intervals

Definition 4.2. A valued binary relation $r$ defined on $A \times A$ is monotone if and only if $\forall x, y r(x, y)$ is non decreasing on $x$ and non increasing on $y$. 
Definition 4.3. Let $x, y, x^{\prime}, y^{\prime}$ be four intervals such that $l\left(x^{\prime}\right)=l(x)+a, u\left(x^{\prime}\right)=$ $u(x)+b, l\left(y^{\prime}\right)=l(y)+c, u\left(y^{\prime}\right)=u(y)+d$ with $a, b, c, d \geq 0$. A valued binary relation $r$ defined on $A \times A$ is monotone if and only if $r\left(x, y^{\prime}\right) \leq r(x, y) \leq r\left(x^{\prime}, y\right)$.

Proposition 4.2. Let $r$ be the valued outranking relation of Definition 4.1, then $r$ is monotone.

Proof : see Annexe (Section 7).

Since we know now that $r$ is reflexive and $S$-strong complete we can try to see if $r$ corresponds to a valued preference structure such as valued total order, valued preorder or valued interval order. We study first of all the $T$-antisymmetricity, necessary condition for valued total orders.

Proposition 4.3. Let $r$ be the valued outranking relation of Definition 4.1, then $r$ is not T-antisymmetric (i.e. there is no t-norm satisfying the T-antisymmetricity).

Proof : see Annexe (Section 7).

Corollary 4.1. Let $r$ be the valued outranking relation of Definition 4.1, then $r$ is not a valued total order.

Preorders, with total orders, are one of the most used preference relations in decision aiding. Their difference from total orders is the fact that they allow the indifference between two different objects, which is not the case with total orders. The remaining property for $r$ being a valued preorder is the transitivity.

Proposition 4.4. Let $r$ be the valued outranking relation of Definition 4.1, then $r$ is not $T$-transitive for any $t$-norm $T$.

Proof : see Annexe (Section 7)

Corollary 4.2. Let $r$ be the valued outranking relation of Definition 4.1, then $r$ is not a valued preorder. 
This result is not surprising. In the crisp case, the preference structures having a numerical representation by intervals, such as semi-orders, interval orders, $P Q I$ interval orders, pseudo orders, are not transitive. Generally the intransitivity of such structures is related to the intransitivity of the symmetric part of the relation (the indifference) which is provided by the cumulation of small indifference thresholds (for more details, see [10, 12, 16, 18]). However, the transitivity of the asymmetric part of the semi orders and interval orders, which are the mostly used ones, remains satisfied, this allowing the choice of the best object without difficulty. Semi orders and interval orders satisfy another property which is interesting for decision aiding. They are Ferrers relations. As we showed in the previous section, there are many ways to define a Ferrers relation in the crisp case and unfortunately the equivalence of these definitions is lost in valued case. However there are some relations between different interpretations, the definition of min-interval order implying all the rest, except one. Our valued outranking relation satisfies this general definition.

Proposition 4.5. Let $r$ be the valued outranking relation of Definition 4.1, then $r$ is a min-interval order.

Proof : see Annexe (Section 7)

As a consequence our interval comparison satisfies the large majority of the definitions of fuzzy interval orders proposed by different authors.

Corollary 4.3. Let $r$ be the valued outranking relation of Definition 4.1, then $r$ satisfies the definitions 2, 3, 4, 6, 7, 8, 9 and 10 of Figure 1. Hence $r$ is a Łukasiewicz-interval order, a weak-interval order, a $T_{L}-S_{L}$-interval order, minmax-interval order and $\lambda$-interval order.

Proof : immediate because of Theorem 3.2

\section{Decomposition of the outranking relation for intervals on $p$ and $i$}

In preference modeling it is very common to separate the outranking relation into two parts in order to differentiate the strict preference from the indifference. The preference is defined as the asymmetric part of the relation while the indifference is considered as its symmetric part. In the crisp case we have: 
$\left\{\begin{array}{l}\forall x, y P(x, y) \Longleftrightarrow R(x, y) \text { and } \operatorname{not}(R(y, x)) \\ \forall x, y I(x, y) \Longleftrightarrow R(x, y) \text { and } R(y, x)\end{array}\right.$

In the valued case, we replace the conjunction operator by a t-norm :

$$
\left\{\begin{array}{l}
\forall x, y p(x, y)=T(r(x, y), N(r(y, x))) \\
\forall x, y i(x, y)=T(r(x, y), r(y, x))
\end{array}\right.
$$

However the choice of the t-norm and the negation may have an important impact and may provide different definitions (for a deep discussion on the subject see [11]).

A classical way is to consider the min operator for the indifference. The negation operation is generally defined as : $N(x)=1-x, \forall x$. However, there is no consensus on the form of preference. In the following we chose to use the same t-norm for $p$ and $i$ :

Definition 5.1. Let $r$ be the valued outranking relation of Definition 4.1, its asymmetric and symmetric parts, denoted respectively by $p$ and $i$ are defined as follows,

$$
\begin{aligned}
& \forall x, y \in A, p(x, y)=\min (r(x, y), 1-r(y, x)) \\
& \forall x, y \in A, i(x, y)=\min (r(x, y), r(y, x))
\end{aligned}
$$

Table 5 presents values of $r, p$ and $i$ depending on the relative positions of $x$ and $y$. The number of cases are the same as the cases presented in Table 2.

\begin{tabular}{|c||c|c|c|c|c|}
\hline Case $(x, y)$ & $r(x, y)$ & $r(y, x)$ & $p(x, y)$ & $p(y, x)$ & $i(x, y)$ \\
\hline \hline 1 & 1 & 0 & 1 & 0 & 0 \\
\hline 2 & 1 & $\frac{u(y)-l(x)}{u(y)-l(y)}$ & $\frac{l(x)-l(y)}{u(y)-l(y)}$ & 0 & $\frac{u(y)-l(x)}{u(y)-l(y)}$ \\
\hline 3 & $\frac{u(x)-l(y)}{u(x)-l(x)}$ & 1 & 0 & $\frac{l(y)-l(x)}{u(x)-l(x)}$ & $\frac{u(x)-l(y)}{u(x)-l(x)}$ \\
\hline 4 & 1 & 1 & 0 & 0 & 1 \\
\hline 5 & 1 & $\frac{u(y)-l(x)}{u(y)-l(y)}$ & $\frac{l(x)-l(y)}{u(y)-l(y)}$ & 0 & $\frac{u(y)-l(x)}{u(y)-l(y)}$ \\
\hline 6 & $\frac{u(x)-l(y)}{u(x)-l(x)}$ & 1 & 0 & $\frac{l(y)-l(x)}{u(x)-l(x)}$ & $\frac{u(x)-l(y)}{u(x)-l(x)}$ \\
\hline 7 & 0 & 1 & 0 & 1 & 0 \\
\hline
\end{tabular}

Table 3: Values of $r, p$ and $i$ depending the relative positions of $x$ and $y$ presented in Table 2 
Remark 5.1. Let $p$ and $i$ be two valued relations of Definition 5.1, then

1. $p$ is defined as the dual of $r: \forall x, y p(x, y)=1-r(y, x)$

2. The semantic of $p$ is the following: there are positive reasons to affirm that $x$ is preferred to $y$ if some points of $y$ are smaller than all the points of $x$. In fact $p$ is defined as:

$$
\forall x, y p(x, y)=\max \left(0, \min \left(1, \frac{l(x)-l(y)}{u(y)-l(y)}\right)\right)
$$

3. $p$ is monotone.

4. $\forall x, y i(x, y)=r(x, y)+r(y, x)-1$

5. $\forall x, y p(x, y)+i(x, y)+p(y, x)=1$

The first remark is a consequence of the fact that for each relative position of $x$ and $y, r(x, y)$ or $r(y, x)$ is equal to one. Concerning the second remark, it is sufficient to analyze the values presented in Table 5 in order to verify the affirmation. The monotonicity of $p$ is a direct consequence of the monotonicity of $r(r(y, x)$ is not decreasing on $y$ and not increasing on $x$, see Proposition 4.2). Remark 4 is also due to the fact that $\forall x, y \max (r(x, y), r(y, x))=1$. The last remark is a direct consequence of Remark 1 and Remark 4.

We will see now if the collection of $p$ and $i$ form a valued preference structure. We begin by presenting the fuzzification of the definition of a crisp preference structure (Definition 2.1).

Definition 5.2 (Fuzzy $\langle P, I\rangle$ preference structure). Let $p$ and $i$ two valued relation defined on $A \times A$, then they form a fuzzy $\langle P, I\rangle$ preference structure iff

(P1) $p$ is irreflexive, $i$ is reflexive

(P2) $p$ is T-asymmetric

(P3) $i$ is symmetric

(P4) $T(p, i)=0$ (exclusivity)

(P5) $S\left(p, S\left(i, p^{-1}\right)\right)=1$ (exhaustivity)

Remark 5.2. For a fuzzy $\langle P, I\rangle$ preference structure, 
1. if $p$ is monotone then for cases 4, 6 and 7 of Table 2 the preference must be zero ;

2. if $p$ is monotone and $p(x, y)=1$ for case 1 of Table 2 and $p(x, y)=0$ for case 2 of Table 2 then we obtain a crisp interval order.

Concerning the first point, the irreflexivity of $p$ implies $p(x, x)=0$ for case 4 and the monotonicity of $p$ implies the rest. For the second point, we get $\forall x, y$ in case $3,4,5,6$ or $7 p(x, y)=0$ because of the monotonicity. By exhaustivity we have $\forall x, y$ in case $2,3,4,5$ or $6 i(x, y)=1$.

For Definition 5.2 the choice of the t-norms and t-conorms is very important. Intuitively we think that for decision aiding it is not suprising to forbid completely the coexistence of $p$ and $p^{-1}$. For this purpose a positive (without zero divisor) t-norm, such as min, can be used. The idea of using a valued relation is introduced in order to allow the coexistence of $p$ and $i$ in some particular cases (for instance if there are some common points to intervals $x$ and $y$ but also some points of $x$ are greater than all the points of $y$, there are some positive reasons for indifference but also preference). For that reason, we think that it is better not to have a positive t-norm for Property P4. We think that Łukasiewicz t-norms can be used for this property. Concerning the exhaustivity, using a positive t-conorm such as max may be very imposing (it means that for each relative position at least one relation $-p$ or $i$ - must be equal to 1 ), the use of Łukasiewicz t-conorms may be a good choice for this property.

Our intuitions are coherent with theoretical results. Van de Walle et al. ([20]) analyzed the use of different type of t-norms and concluded their paper by suggesting the use of Łukasiewicz t-norms. Their analysis concerned preference structures including the incomparability but their results remain valid in the case of fuzzy $\langle P, I\rangle$ preference structure. They showed that with positive t-norms only crisp preference structures can be defined.

Proposition 5.1 ([20]). Consider a de Morgan triplet $M$ with a positive t-norm (without zero divisor). If $M$ is used in all the properties of Definition 5.2 then the valued relations $p$ and $i$ are all crisp.

Hence t-norms having zero divisors must be used in order to allow valued relations. However the following proposition shows that some type of t-norms with zero divisors are not very intuitive for preference modeling since they do not allow the use of the whole range $[0,1]$. 
Proposition 5.2 ([20]). Consider a de Morgan triplet $M$ with a continuous nonArchimedean t-norm with zero divisors. If $M$ is used in all the properties of Definition 5.2 then $\exists x_{\varsigma} \in[0,1[$ such that $\forall x, y$,

$$
\begin{aligned}
p(x, y)<1 & \Longrightarrow p(x, y)<x_{\varsigma}, \\
i(x, y)<1 & \Longrightarrow i(x, y)<x_{\varsigma} .
\end{aligned}
$$

In the light of these two propositions Van de Walle et al. proposed the use of Łukasiewicz triplet in Definition 5.2:

Definition 5.3 (Lukasiewicz $\langle P, I\rangle$ preference structure [20]). Let $p$ and $i$ be two valued relations defined on $A \times A$, then they form a Łukasiewicz $\langle P, I\rangle$ preference structure iff

$(P 1) p$ is irreflexive, $i$ is reflexive

(P2) p is $T_{L}$-asymmetric

(P3) i is symmetric

(P4) $T_{L}(p, i)=0$

$(P 5) S_{L}\left(p, S_{L}\left(i, p^{-1}\right)\right)=1$

Proposition 5.3. Let $p$ and $i$ be the valued relations of Definition 5.1, then they form a Łukasiewicz $\langle P, I\rangle$ preference structure.

Proof : see Annexe (Section 7)

However as we mentioned before we think that in some contexts it may be preferable to use the $T_{\min }$ for Property 2. Hence we propose a restriction in the definition given by Van de Walle et al.:

Definition 5.4 (Mixt Fuzzy $\langle P, I\rangle$ preference structure). Let $p$ and $i$ be two valued relations defined on $A \times A$, then they form a mixt Fuzzy $\langle P, I\rangle$ preference structure iff

$(P 1)$ p is irreflexive, $i$ is reflexive 
(P2) p is $T_{m i n}$-asymmetric

(P3) $i$ is symmetric

(P4) $T_{L}(p, i)=0$ (exclusivity)

$(P 5) S_{L}\left(p, S_{L}\left(i, p^{-1}\right)\right)=1$ (exhaustivity)

Proposition 5.4. Let $p$ and $i$ be the valued relations of Definition 5.1, then they form a mixt Fuzzy $\langle P, I\rangle$ preference structure.

Proof : see Annexe (Section 7)

Our relations $p$ and $i$ show that the change of Property 2 in Definition 5.3 has not a negative affect since the relations $p$ and $i$ can be valued and their evaluation makes use of the whole range $[0,1]$.

Proposition 5.5. Let $p$ and $i$ two valued relation of Definition 5.4, then they can be valued and take values in the whole range $[0,1]$.

Proof : see Annexe (Section 7)

We now know that our relations $p$ and $i$ form a valued preference structure. However this structure is neither a valued total order nor a valued preorder since $r$ is not $T$-transitive. We mentioned in the previous section that in the crisp case even if interval orders are not transitive their asymmetric part is transitive. We have also the satisfaction of the transitivity with our valued preference relation.

Proposition 5.6. Let $p$ be the valued outranking relation of Definition 5.1, then $p$ is $T_{\text {min }}$-transitive.

Proof : see Annexe (Section 7)

Corollary 5.1. Let $p$ be the valued binary relation of Definition 5.1, then $p$ is T-transitive with any t-norm.

Proof: see Annexe (Section 7)

We saw that $p$ and $i$ have some nice properties for preference modeling. By Corollary 4.3 we know that $p{ }^{{ }} T_{L} i{ }^{\circ} T_{L} p \subseteq p$ (they correspond to a weak interval order since). We will analyse in the fallowing if the condition $p \circ_{T_{\min }} i \circ_{T_{\min }} p \subseteq p$ (necessary condition for strong-interval orders) is verified with our relations. 
Proposition 5.7. Let $p$ and $i$ be two valued relations of Definition 5.1, then they do not form a strong-interval order.

Proof: see Annexe (Section 7)

In a more general way when some intuitive conditions are imposed to the relations the condition $p \circ_{T} i \circ_{T} p \subseteq p$ is not verified with positive t-norms. We will impose three intuitive conditions :

- the monotonicity of $p$

- the fact that $p(x, y)$ is different from zero if $l(x)>l(y)$ and $u(x)>u(y)$. This situation corresponds to the case 2 of Table 2 and we know that if $p(x, y)=0$ in Case 2 we have crisp interval orders, see point 2 of Remark 5.2

- the fact that indifference is different from zero when two intervals have some points in common

Proposition 5.8. If $p$ is monotone and $p(x, y)>0$ for case 2 of Table 2 and $i(x, y)>0$ when $x$ and $y$ have some common points then $p \circ_{L} i \circ_{L} p \subseteq p$ can not be verified with positive t-norms.

Proof: see Annexe (Section 7)

\section{Conclusion}

In this paper we proposed a valued outranking relation for interval comparison. Our relation has the advantage of being intuitive, it satisfies important properties for decision making such as the monotonicity and corresponds to a general valued Ferrers relation in the sense that it satisfies the majority of definitions using different de Morgan triplets. Our analysis allowed us to point out also some existing results on the characterization of valued interval orders. We presented the difficulties of obtaining a unique definition for these structures and showed the relations between different definitions proposed in the literature. We then divided our outranking relation into two in order to differentiate the preference from the indifference. We showed that our separation is meaningful for preference modeling since it corresponds to a valued preference structure. It satisfies nice properties such as the monotonicity and the transitivity of the valued preference relation. 
Concluding our study we realized that one of the definition of a valued interval order $\left(p \circ_{T_{\min }} i{ }_{T_{\text {min }}} p \subseteq p\right)$ is not coherent with some intuitive conditions, hence we do not recommend its use for decision making.

For future studies, a numerical representation of valued interval orders is not studied in the literature. Our outranking relation can be a starting point for this study. On the other hand, studies similar to ours are missing for other preference structures using interval representation. We think that semiorders, $P Q I$ interval orders and pseudo-orders deserve also such analysis.

\section{References}

[1] G. Bosi, J.C. Candeal, M.J. Campiòn, and E. Indurain. Interval-valued representability of qualitative data: the continuous case. International Journal of Uncertainty, Fuzziness and Knowledge-Based Systems, 15:299-319, 2007.

[2] G. Bosi, J. Gutierrez Garca, and E. Indurain. Unified representability of total preorders and interval orders through a single function: The lattice approach. Order, 26:255-275, 2009.

[3] D. Bouyssou and Ph. Vincke. Binary relations and preference modeling. In D. Bouyssou, D.Dubois, M. Pirlot, and H. Prade, editors, Decision-making Process Concepts and Methods, pages 49-84. ISTE / Wiley, 2009.

[4] D. S. Bridges and G. B. Mehta. Representations of Preference Orderings. Springer-Verlang, Berlin, 1995.

[5] A. Bufardi. An alternative definition for fuzzy interval orders. Fuzzy Sets and Systems, 133:249-259, 2003.

[6] B. De Baets, E. Kerre, and B. Van De Walle. Fuzzy preference structures and their characterization. Journal of Fuzzy Mathematics, 3:373, 1995.

[7] B. De Baets and B. Van De Walle. Weak and strong fuzzy interval orders. Fuzzy Sets and Systems, 79:213-225, 1996.

[8] S. Díaz, B. De Baets, and S. Montes. On the Ferrers property of valued interval orders. TOP, 19:421-447, 2011.

[9] D. Dubois and H. Prade. Fuzzy sets and systems - Theory and applications. Academic press, New York, 1980. 
[10] P.C. Fishburn. Interval Orders and Interval Graphs. J. Wiley, New York, 1985.

[11] J. Fodor and M. Roubens. Fuzzy preference modelling and multicriteria decision support. Kluwer Academic Publishers, 1994.

[12] R.D. Luce. Semiorders and a theory of utility discrimination. Econometrica, 24:178-191, 1956.

[13] L. Nachbin. Topology and Order. Van Nostrand Reinhold, New York, 1965.

[14] S.N. Ovchinnikov. Modelling valued preference relation. In J. Kacprzyk and M. Fedrizzi, editors, Multiperson decion making using fuzzy sets and possibility theory, pages 64-70. Kluwer, Dordrecht, 1990.

[15] M. Öztürk, A. Tsoukiàs, and Ph. Vincke. Preference modelling. In M. Ehrgott, S. Greco, and J. Figueira, editors, Multiple Criteria Decision Analysis: State of the Art Surveys, pages 27-73. Springer, 2005.

[16] M. Pirlot and Ph. Vincke. Semi Orders. Kluwer Academic, Dordrecht, 1997.

[17] M. Roubens and Ph. Vincke. Preference Modelling. LNEMS 250, Springer Verlag, Berlin, 1985.

[18] B. Roy. Multicriteria Methodology for Decision Aiding. Kluwer Academic, Dordrecht, 1996.

[19] B. Schweizer and A. Sklar. Probabilistic Metric Spaces. Elsevier Science, New York, 1983.

[20] B. Van De Walle, B. De Baets, and E. Kerre. A plea for the use of lukasiewicz triplets in the definition of fuzzy preference structures.(i). general argumentation. Fuzzy Sets and Systems, 97:349-359, 1998.

\section{Annexe}

\section{Proof of Theorem 3.2.}

We make use of some results of the papers $[5,7]$ and we add some counterexamples in order to complete the proof. 
Equivalences:

$1 \Longleftrightarrow 2$ : theorem 4.4 of [7],

$1 \Longleftrightarrow 3$ : proposition 4.6 of [7],

$1 \Longleftrightarrow 4$ : theorem 6.1 of [7],

$6 \Longleftrightarrow 7 \Longleftrightarrow 8 \Longleftrightarrow 9$ : proposition 4.3 of [6] and theorem 5.2 of [7].

Implication:

$1 \Longrightarrow 6$ : proposition 4.3 of [7],

$5 \Longrightarrow 7:$ theorem 5.4 of [7],

$9 \Longrightarrow 10$ : theorem 5.2 of [7].

Not implication:

$10 \nRightarrow 6$ : counter example 5.2 of [7],

$1 \nRightarrow 5$ : remark 5.4 of [5],

$5 \nRightarrow 2$ : since there is not any counter example for this case, we propose a simple one. Let $x, y, z, t$ be four elements of $A$ and $p$ and $i$ two valued binary relations where $\forall x, y i(x, y)$ iff $N(p(x, y))$ and $N(p(y, x))$. We propose $p(x, y)=$ $0.9, p(z, t)=0.9, p(x, t)=0.5, p(z, y)=0.6$ and $i(z, y)=i(y, z)=0.4$.

Let us remark that we considered here a Łukasiewicz preference structure and our relations verify the properties of such structure. The use of Zadeh t-nom and tconorm for the definition of a fuzzy preference structure provides crisp relations. It is easy to see that $T_{\min }\left(p(x, y), T_{\min }(i(y, z), p(z, t))=0.4<p(x, t)\right.$. However $T_{\min }(p(x, y), p(z, t))>S_{\max }(p(x, t), p(z, y))$. So the assertion 5 is verified while the assertion 2 is not.

$6 \nRightarrow 1: 5 \Longrightarrow 6$ and $5 \nRightarrow 1$ hence $6 \nRightarrow 1$.

$10 \nRightarrow 5$ : since there is not any counter example for this case, we propose a simple one. Let $x, y, z, t$ be four elements of $A$ and $r$ a valued binary relation such that: $p(x, y)=0.3, i(y, z)=0.4, p(z, t)=0.4$ and $p(x, t)=0.2$. It is easy to see that $T_{L}\left(p(x, y), T_{L}(i(y, z), p(z, t))\right)=0$ so $T_{L}\left(p(x, y), T_{L}(i(y, z), p(z, t))\right)<$ $p(x, t)$. But on the other hand we have $\min (p(x, y), \min (i(y, z), p(z, t)))=0.3$ hence $\min (p(x, y), \min (i(y, z), p(z, t))) \not \leq p(x, t)$. So the assertion 10 is verified while the assertion 5 is not.

\section{Proof of Proposition 4.1.}

- $r$ is reflexive : immediate.

- $r$ is $S$-strong complete : immediate since $\forall x, y, \max (r(x, y), r(y, x))=1$ (see Table 2). 


\section{Proof of Proposition 4.2.}

$r$ is monotone: Consider

$x^{\prime}$ such that $u\left(x^{\prime}\right)=u(x)+a$ and $l\left(x^{\prime}\right)=l(x)+b$ with $a, b \in \mathbb{R}^{+}$and $y^{\prime}$ such that $u\left(y^{\prime}\right)=u(y)+a^{\prime}$ and $l\left(y^{\prime}\right)=l(y)+b^{\prime}$ with $a^{\prime}, b^{\prime} \in \mathbb{R}^{+}$.

We will show that $r\left(x, y^{\prime}\right) \leq r(x, y) \leq r\left(x^{\prime}, y\right)$ in Table 2.

- $r(x, y) \leq r\left(x^{\prime}, y\right)$ : We consider different relative positions of $x$ and $y$ shown

- $x$ and $y$ in Case $1: r\left(x^{\prime}, y\right)$ corresponds to the case 1 and $r\left(x^{\prime}, y\right)=1$.

- $x$ and $y$ in Case $2: r\left(x^{\prime}, y\right)$ corresponds to the cases 1 or 2 and $r\left(x^{\prime}, y\right)=1$.

- $x$ and $y$ in Case 3: $r(x, y)=\frac{u(x)-l(y)}{u(x)-l(x)}$ and $r\left(x^{\prime}, y\right)$ corresponds to the cases 1 or 2 or 3 . If $x^{\prime}$ and $y$ are in case 1 or case 2 then $r\left(x^{\prime}, y\right)=1$. If they are in case 3 then $r\left(x^{\prime}, y\right)=\frac{u(x)-l(y)+a}{u(x)-l(x)+(a-b)}$. Since $a, b \in \mathbb{R}^{+}$and $u(x)-l(y) \leq u(x)-l(x)$, we have $r(x, y) \leq r\left(x^{\prime}, y\right)$.

- $x$ and $y$ in Case $4: r\left(x^{\prime}, y\right)$ corresponds to the cases 1 or 2 and $r\left(x^{\prime}, y\right)=1$.

- $x$ and $y$ in Case $5: r\left(x^{\prime}, y\right)$ corresponds to the cases 1 or 2 or 5 . Hence, $r\left(x^{\prime}, y\right)=1$.

- $x$ and $y$ in Case $6: r(x, y)=\frac{u(x)-l(y)}{u(x)-l(x)}$ and $r\left(x^{\prime}, y\right)$ corresponds to the cases $1,2,3,4,5$ or 6 . If $x^{\prime}$ and $y$ are in case $1,2,4$ or case 5 then $r\left(x^{\prime}, y\right)=1$. If they are in case 3 or 6 then $r\left(x^{\prime}, y\right)=\frac{u(x)-l(y)+a}{u(x)-l(x)+(a-b)}$. Since $a, b \in \mathbb{R}^{+}$and $u(x)-l(y) \leq u(x)-l(x)$, we have $r(x, y) \leq r\left(x^{\prime}, y\right)$.

- $x$ and $y$ in Case $7: r(x, y)$ being equal to zero, $r(x, y) \leq r\left(x^{\prime}, y\right)$.

We show that for all the initial relative positions of $x$ and $y, r(x, y) \leq r\left(x^{\prime}, y\right)$.

- $r\left(x, y^{\prime}\right) \leq r(x, y)$ : We reconsider different relative positions of $x$ and $y$ shown in Table 2.

- $x$ and $y$ in Case 1 or 2 or 4 or 5: $r(x, y)=1$. 
- $x$ and $y$ in Case $3: r(x, y)=\frac{u(x)-l(y)}{u(x)-l(x)}$ and $r\left(x, y^{\prime}\right)$ corresponds to the cases 3 or 6 or 7. If $x$ and $y^{\prime}$ are in case 7 then $r\left(x, y^{\prime}\right)=0$. If they are in case 3 or 6 then $r\left(x, y^{\prime}\right)=\frac{u(x)-\left(l(y)+a^{\prime}\right)}{u(x)-l(x)}$. Since $a^{\prime} \in \mathbb{R}^{+}$we have $r\left(x, y^{\prime}\right) \leq r(x, y)$.

- $x$ and $y$ in Case $6: r(x, y)=\frac{u(x)-l(y)}{u(x)-l(x)}$ and $r\left(x, y^{\prime}\right)$ corresponds to the cases 6 or 7. If $x$ and $y^{\prime}$ are in case 7 then $r\left(x, y^{\prime}\right)=0$. If they are in case 6 then $r\left(x, y^{\prime}\right)=\frac{u(x)-\left(l(y)+a^{\prime}\right)}{u(x)-l(x)}$. Since $a^{\prime} \in \mathbb{R}^{+}$we have $r\left(x, y^{\prime}\right) \leq r(x, y)$.

- $x$ and $y$ in Case $7: r\left(x, y^{\prime}\right)$ being equal to zero, $r\left(x, y^{\prime}\right) \leq r(x, y)$.

We show that for all the initial relative positions of $x$ and $y, r\left(x, y^{\prime}\right) \leq r(x, y)$.

As a conclusion $r$ is monotone.

\section{Proof of Proposition 4.3:}

We show that $\exists x, y$, with $x \neq y$ where $T(r(x, y), r(y, x)) \neq 0$. Let $x$ and $y$ be two intervals defined on $A$ such that $l(x)=l(y)<u(y)<u(x)$. Then we have $r(x, y)=r(y, x)=1$ which implies $T(r(x, y), r(y, x))=1$.

\section{Proof of Proposition 4.4.}

$r$ is not $T$-transitive : A counter-example proving that $\exists x, y, z$ such that $T(r(x, y), r(y, z))>r(x, z)$

Consider three intervals $x, y$ and $z$ with $l(y)<l(x)<u(x)<l(z)<u(z)<$ $u(y)$. Then we have $r(x, y)=1,0<r(y, z)<1$ and $r(x, z)=0$. On the other hand we know that $T(r(x, y), r(y, z))=r(y, z)$ since $\forall x T(1, x)=x$. Hence $T(r(x, y), r(y, z))=r(y, z)>r(x, z)$.

\section{Proof of Proposition 4.5:}

We know that $r$ is reflexive and $S$-strong complete (see Proposition 4.1). We will prove that $R$ is $T_{\min }-S_{\max }$-Ferrers:

$$
\forall x, y, z, t, T_{\min }(r(x, y), r(z, t)) \leq S_{\max }(r(x, t), r(z, y))
$$


The inequality is directly verified when $r(x, t)=1$ or $r(z, y)=1$ (cases: 1,2 , 4 and 5 of Table 2). Hence, in the following we will analyze the situations where $r(x, t) \neq 1$ and $r(z, y) \neq 1$ (cases 3, 6, 7 of the Table 2). There are 9 situations :

$$
\begin{aligned}
& r(x, t) \neq 1 \Longleftrightarrow\left\{\begin{array}{l}
l(x)<u(x)<l(t)<u(t) \text { (case } 7 \text { of table } 2), \\
\text { or } \\
l(x)<l(t)<u(t)<u(x) \text { (case } 3 \text { of table } 2), \\
\text { or } \\
l(x)<l(t)<u(x)<u(t) \text { (case } 6 \text { of table } 2) ;
\end{array}\right. \\
& r(z, y) \neq 1 \Longleftrightarrow\left\{\begin{array}{l}
l(z)<u(z)<l(y)<u(y) \text { (case } 7 \text { of table } 2), \\
\text { or } \\
l(z)<l(y)<u(y)<u(z) \text { (case } 3 \text { of table } 2), \\
\text { or } \\
l(z)<l(y)<u(z)<u(y) \text { (case } 6 \text { of table } 2) .
\end{array}\right.
\end{aligned}
$$

1. Case 7 of Table 2 for $(x, t): l(x)<u(x)<l(t)<u(t)$ (Inequation $A$ ) and Case 7 of Table 2 for $(z, y): l(z)<u(z)<l(y)<u(y)$ (Inequation $B$ )

We analyze two cases:

$-r(x, y)=0:$ trivial

- $r(x, y) \neq 0$ : we will show that if $r(x, y) \neq 0$ then $r(z, t)=0$.

We have $u(z)<l(y)$ (Ineq. $B$ ), $l(y)<u(x)$ (since $r(x, y) \neq 0)$ and $u(x)<l(t)$ (Ineq.A). Then, we get $u(z)<l(t)$ implying $r(z, t)=0$. Hence we get $\min (r(x, y), r(z, t))=0$.

As a conclusion Inequility 1 is satisfied in this case.

2. Case 3 of Table 2 for $(x, t): l(x)<l(t)<u(t)<u(x)$ and

Case 3 of Table 2 for $(z, y): l(z)<l(y)<u(y)<u(z))$.

We analyze two cases:

- If $l(t) \geq l(y)$ then $l(t) \geq l(y)>l(z)$. This implies :

$$
r(z, t)=\max \left(0, \frac{u(z)-l(t)}{u(z)-l(z)}\right)
$$

because

- if $l(t)>u(z)$ then $r(z, t)=0$ (case 7 of Table 2) 
- if $l(t) \leq u(z)$ then the position of the intervals $z$ and $t$ will be case 6 or case 3 of Table 2, hence $r(z, t)=\frac{u(z)-l(t)}{u(z)-l(z)}$.

so we get

$$
r(z, y)=\frac{u(z)-l(y)}{u(z)-l(z)} \text { and } r(z, t)=\max \left(0, \frac{u(z)-l(t)}{u(z)-l(z)}\right)
$$

which gives $r(z, y) \geq r(z, t)$.

In conclusion, we get: $\min (r(x, y), r(z, t)) \leq \max (r(x, t), r(z, y))$.

Similar reasoning are used in all the remaining cases for which we will not detail this part.

- If $l(t)<l(y)$ then $l(y)>l(t)>l(x)$

$$
r(x, t)=\frac{u(x)-l(t)}{u(x)-l(x)} \text { and } r(x, y)=\max \left(0, \frac{u(x)-l(y)}{u(x)-l(x)}\right)
$$

which gives $r(x, t)>r(x, y)$.

In conclusion, we get: $\min (r(x, y), r(z, t)) \leq \max (r(x, t), r(z, y))$.

As a conclusion the Inequility 1 is satisfied in this case.

3. Case 3 of Table 2 for $(x, t): l(x)<l(t)<u(t)<u(x)$ and

Case 6 of Table 2 for $(z, y): l(z)<l(y)<u(z)<u(y)$.

- If $l(t)>l(y)$ then $l(t)>l(y)>l(z)$

$$
r(z, y)=\frac{u(z)-l(y)}{u(z)-l(y)} \text { and } r(z, t)=\max \left(0, \frac{u(z)-l(t)}{u(z)-l(z)}\right)
$$

which gives $r(z, y)>r(z, t)$.

In conclusion, we get: $\min (r(x, y), r(z, t)) \leq \max (r(x, t), r(z, y))$.

- If $l(t) \leq l(y)$ then $l(y) \geq l(t)>l(x)$

$$
r(x, t)=\frac{u(x)-l(t)}{u(x)-l(x)} \text { and } r(x, y)=\max \left(0, \frac{u(x)-l(y)}{u(x)-l(x)}\right)
$$


which gives $r(x, t) \geq r(x, y)$.

In conclusion, we get: $\min (r(x, y), r(z, t)) \leq \max (r(x, t), r(z, y))$.

As a conclusion the Inequility 1 is satisfied in this case.

4. Case 6 of Table 2 for $(x, t): l(x)<l(t)<u(x)<u(t)$ and

Case 3 of Table 2 for $(z, y): l(z)<l(y)<u(y)<u(z)$

The case being symmetric to the previous one, the proof is immediate.

5. Case 3 of Table 2 for $(x, t): l(x)<l(t)<u(t)<u(x)$ and

Case 7 of Table 2 for $(z, y): l(z)<u(z)<l(y)<u(y)$.

- If $l(t)>l(y)$ then $l(t)>l(y)>u(z)$.

We have $(l(t)>u(z)) \longrightarrow r(z, t)=0$,

In conclusion we get: $\min (r(x, y), r(z, t)) \leq \max (r(x, t), r(z, y))$.

- If $l(t) \leq l(y)$ then $l(y) \geq l(t)>l(x)$

$$
r(x, t)=\frac{u(x)-l(t)}{u(x)-l(x)} \text { and } r(x, y)=\max \left(0, \frac{u(x)-l(y)}{u(x)-l(x)}\right)
$$

which gives $r(x, t) \geq r(x, y)$.

In conclusion, we get: $\min (r(x, y), r(z, t)) \leq \max (r(x, t), r(z, y))$.

As a conclusion the Inequility 1 is satisfied in this case.

6. Case 7 of Table 2 for $(x, t): l(x)<u(x)<l(t)<u(t)$ and Case 3 of Table 2 for $(z, y): l(z)<l(y)<u(y)<u(z)$.

The case being symmetric to the previous one, the proof is immediate.

7. Case 6 of Table 2 for $(x, t): l(x)<l(t)<u(x)<u(t)$ and Case 6 of Table 2 for $(z, y): l(z)<l(y)<u(z)<u(y)$.

- If $l(t) \geq l(y)$ then $l(t) \geq l(y)>l(z)$

$$
r(z, y)=\frac{u(z)-l(y)}{u(z)-l(z)} \text { and } r(z, t)=\max \left(0, \frac{u(z)-l(t)}{u(z)-l(z)}\right)
$$


which gives $r(z, y) \geq r(z, t)$.

In conclusion, we get: $\min (r(x, y), r(z, t)) \leq \max (r(x, t), r(z, y))$.

- If $l(t)<l(y)$ then $l(y)>l(t)>l(x)$

$$
r(x, t)=\frac{u(x)-l(t)}{u(x)-l(x)} \text { and } r(x, y)=\max \left(0, \frac{u(x)-l(y)}{u(x)-l(x)}\right)
$$

which gives $r(x, t)>r(x, y)$.

In conclusion, we get: $\min (r(x, y), r(z, t)) \leq \max (r(x, t), r(z, y))$.

Hence, the Inequility 1 is satisfied in this case.

8. Case 6 of Table 2 for $(x, t): l(x)<l(t)<u(x)<u(t)$ and

Case 7 of Table 2 for $(z, y): l(z)<u(z)<l(y)<u(y)$.

- If $l(t) \geq l(y)$ then $l(t) \geq l(y)>u(z)$

We have $l(t)>u(z) \longrightarrow r(z, t)=0$.

In conclusion, we get: $\min (r(x, y), r(z, t)) \leq \max (r(x, t), r(z, y))$.

- If $l(t)<l(y)$ then $l(y)>l(t)>l(x)$

$$
r(x, t)=\frac{u(x)-l(t)}{u(x)-l(x)} \text { and } r(x, y)=\max \left(0, \frac{u(x)-l(y)}{u(x)-l(x)}\right)
$$

which gives $r(x, t)>r(x, y)$.

In conclusion, we get: $\min (r(x, y), r(z, t)) \leq \max (r(x, t), r(z, y))$.

Hence, the Inequility 1 is satisfied in this case.

9. Case 7 of Table 2 for $(x, t): l(x)<u(x)<l(t)<u(t)$ and Case 6 of Table 2 for $(z, y): l(z)<l(y)<u(z)<u(y)$

The case being symmetric to the previous one, the proof is immediate. 
Thus, $\forall x, y, z, t \min (r(x, y), r(z, t)) \leq \max (r(x, t), r(z, y))$.

\section{Proof of Proposition 5.3}

- $p$ is irreflexive, $i$ is reflexive : obvious.

- $p$ is $T_{L}$-asymmetric : we show that $p$ is $T$-asymmetric with any t-norm. $\forall x, y, p(x, y)$ or $p(y, x)$ is equal to zero hence $\forall x, y, T(p(x, y), p(y, x))=$ 0 .

- $i$ is symmetric : obvious

- $T_{L}(p, i)=0$. We showed that $\forall x, y p(x, y)=1-r(y, x)$ and $i(x, y)=$ $r(x, y)+r(y, x)-1$. Hence, $T_{L}(p(x, y), i(x, y))=\max (p(x, y)+i(x, y)-$ $1,0)=\max (r(x, y)-1,0)=0$

- $S_{L}\left(p, S_{L}\left(i, p^{-1}\right)\right)=1$. Point 5 of Remark 5.1 shows that $\forall x, y p(x, y)+$ $i(x, y)+p(y, x)=1$. Hence $S_{L}\left(p, S_{L}\left(i, p^{-1}\right)\right)=1$.

\section{Proof of Proposition 5.4}

Immediate since we showed in the proof of Property 5.3 that $p$ is $T$-asymmetric with any t-norm.

\section{Proof of Proposition 5.5}

Immediate since our relations $p$ and $i$ satisfy the conditions.

\section{Proof of Proposition 5.6}

$p$ is $T_{\min }$-transitive : we will show that $\forall x, y, z T_{\min }(p(x, y), p(y, z)) \leq p(x, z)$.

The inequality is directly verified when $p(x, y)=0$ or $p(y, z)=0$ (cases: 3, 4, 6 and 7 of Table 2). Hence, in the following we will analyze the situations where $p(x, y) \neq 0$ and $p(y, z) \neq 0$ (cases 1,2 and 5 of the Table 2). There are 9 situations : 


$$
\begin{aligned}
& p(x, y) \neq 0 \Longleftrightarrow\left\{\begin{array}{l}
l(y)<u(y)<l(x)<u(x) \text { (case } 1 \text { of table } 2), \\
\text { or } \\
l(y)<l(x)<u(y)<u(x) \text { (case } 2 \text { of table } 2), \\
\text { or } \\
l(y)<l(x)<u(x)<u(y) \text { (case } 5 \text { of table } 2) ;
\end{array}\right. \\
& p(y, z) \neq 0 \Longleftrightarrow\left\{\begin{array}{l}
l(z)<u(z)<l(y)<u(y) \text { (case } 1 \text { of table 2), } \\
\text { or } l(z)<l(y)<u(z)<u(y) \text { (case } 2 \text { of table 2), } \\
\text { or } \\
l(z)<l(y)<u(y)<u(z) \text { (case } 5 \text { of table 2); }
\end{array}\right.
\end{aligned}
$$

1. Case 1 of Table 2 for $(x, y): l(y)<u(y)<l(x)<u(x)$ and Case 1 of Table 2 for $(y, z): l(z)<u(z)<l(y)<u(y)$.

We have directly $u(z)<l(x)$ which implies $p(x, z)=1$

2. Case 1 of Table 2 for $(x, y): l(y)<u(y)<l(x)<u(x)$ and Case 2 of Table 2 for $(y, z): l(z)<l(y)<u(z)<u(y))$.

We have directly $u(z)<l(x)$ which implies $p(x, z)=1$

3. Case 1 of Table 2 for $(x, y): l(y)<u(y)<l(x)<u(x)$ and Case 5 of Table 2 for $(y, z): l(z)<l(y)<u(y)<u(z))$.

$p(y, z)=\frac{l(y)-l(z)}{u(z)-l(z)}$ and $p(x, z)=\min \left(\frac{l(x)-l(z)}{u(z)-l(z)}, 1\right)$

which gives $p(x, z) \geq p(y, z)$. In fact $p(x, y)$ can be in case 1,2 or 5 . In case 1 we have $p(x, z)=1$ and in case 2 or 5 we have $p(x, z)=\frac{l(x)-l(z)}{u(z)-l(z)}$. We use similar reasoning in all the fallowing cases where we do not detail this part.

In conclusion, we get: $\min (p(x, y), p(y, z)) \leq p(x, z)$.

4. Case 2 of Table 2 for $(x, y): l(y)<l(x)<u(y)<u(x)$ and Case 1 of Table 2 for $(y, z): l(z)<u(z)<l(y)<u(y)$.

We have directly $u(z)<l(x)$ which implies $p(x, z)=1$

5. Case 2 of Table 2 for $(x, y): l(y)<l(x)<u(y)<u(x)$ and Case 2 of Table 2 for $(y, z): l(z)<l(y)<u(z)<u(y)$. 
$p(y, z)=\frac{l(y)-l(z)}{u(z)-l(z)}$ and $p(x, z)=\min \left(\frac{l(x)-l(z)}{u(z)-l(z)}, 1\right)$

which gives $p(x, z) \geq p(y, z)$.

In conclusion, we get: $\min (p(x, y), p(y, z)) \leq p(x, z)$.

6. Case 2 of Table 2 for $(x, y): l(y)<l(x)<u(y)<u(x)$ and

Case 5 of Table 2 for $(y, z): l(z)<l(y)<u(z)<u(y)$.

$p(y, z)=\frac{l(y)-l(z)}{u(z)-l(z)}$ and $p(x, z)=\min \left(\frac{l(x)-l(z)}{u(z)-l(z)}, 1\right)$

which gives $p(x, z) \geq p(y, z)$.

In conclusion, we get: $\min (p(x, y), p(y, z)) \leq p(x, z)$.

7. Case 5 of Table 2 for $(x, y): l(y)<l(x)<u(x)<u(y)$ and

Case 1 of Table 2 for $(y, z): l(z)<u(z)<l(y)<u(y)$.

We have directly $u(z)<l(x)$ which implies $p(x, z)=1$

8. Case 5 of Table 2 for $(x, y): l(y)<l(x)<u(x)<u(y)$ and Case 2 of Table 2 for $(y, z): l(z)<l(y)<u(z)<u(y)$.

$p(y, z)=\frac{l(y)-l(z)}{u(z)-l(z)}$ and $p(x, z)=\min \left(\frac{l(x)-l(z)}{u(z)-l(z)}, 1\right)$

which gives $p(x, z) \geq p(y, z)$.

In conclusion, we get: $\min (p(x, y), p(y, z)) \leq p(x, z)$.

9. Case 5 of Table 2 for $(x, y): l(y)<l(x)<u(x)<u(y)$ and

Case 5 of Table 2 for $(y, z): l(z)<l(y)<u(y)<u(z)$.

$p(y, z)=\frac{l(y)-l(z)}{u(z)-l(z)}$ and $p(x, z)=\frac{l(x)-l(z)}{u(z)-l(z)}$

which gives $p(x, z) \geq p(y, z)$.

In conclusion, we get: $\min (p(x, y), p(y, z)) \leq p(x, z)$.

Thus, $\forall x, y, z \min (p(x, y), p(y, z)) \leq p(x, z)$

\section{Proof of Corollary 5.1}

The proof is immediate since the Zadeh t-norm $\left(T_{\min }\right)$ is the pointwise largest t-norm, i.e. for any t-norm $T$ and $\forall a, b \in[0,1] T(a, b) \leq T_{\min }(a, b)$.

\section{Proof of Proposition 5.7:}


Here is a counter-example that proves $p \circ_{T_{\min }} i \circ_{T_{\min }} p \nsubseteq p$ : Let $x, y z$ and $t$ be four intervals such that

i. $u(x)>u(y)>u(z)>u(t)$

ii. $l(z)>l(t)>l(x)>l(y)$

iii. $l(z)>u(t)$

Then we have $p(x, y)>0$ (case 2 of Table 2), $i(y, z)>0$ (case 3 of Table 2) and $p(z, t)=1$ (case 1 of Table 2 ), thus

$\left(p \circ \circ_{T_{\min }} i \circ p\right)(x, z)=\min (p(x, y), i(y, z), p(z, t))>0$ (case 3 of Table 2$)$, but $p(x, t)=0$, thus

$\min \{p(x, y), \min (i(y, z), p(z, t))\}>p(x, t)$.

\section{Proof of Proposition 5.8:}

Let $x, y z$ and $t$ be four intervals such that

$$
l(y)<l(x)<l(t)<l(z)<u(y)<u(x)<u(t)<u(z)
$$

then we have $p(x, y)>0$ and $p(z, t)>0$ since they are in case 2 of Table 2. $i(y, z)>0$ since $(y, z)$ are in case 6 . And $p(x, t)=0$ since $p$ is monotone and $x$ and $t$ are in case 6 of Table 2 (see Remark 5.2). Hence $T(p(x, y), T(i(y, z), p(z, t))) \leq$ 0 can not be verified with positive t-norms. 